\title{
Eritema multiforme induzido por hidroxicloroquina: relato de caso
}

\author{
Case report: Erythema multiforme associated with the use of hydroxychloroquine
}

João Pedro Araújo Bruno ${ }^{\dagger}$, Marise Maleck ${ }^{\dagger, *} \S^{*}$, Maria Olivia de Lima Bezerra ${ }^{\dagger}$

Como citar esse artigo. Bruno,

J.P.A.; Maleck, M.; Bezerra, M.O.L.

Eritema multiform induzido por

hidroxicloroquina: relato de caso. Revista

de Saúde. 2020 Jan./Jun.; 11 (1): 02-04.

\author{
Resumo
}

As farmacodermias possuem uma incidência em torno de 5 a $30 \%$, dentre 3 a $6 \%$ de todas as admissões hospitalares. As reações medicamentosas adversas (RMA) estão entre as principais causas de morte em vários países. O objetivo deste estudo foi relatar o caso de uma paciente com quadro clínico de Eritema Multiforme após o uso de Hidroxicloroquina em terapia para Chikungunya (CHIKV). O caso evoluiu para um exantema generalizado. Após a remissão das lesões cutâneas, foi diagnosticado como Artrite Reumatóide (AR) soronegativa. Prosseguiu-se a terapêutica com corticoide e metrotexa-to com significativa melhora do quadro. Este estudo concluiu a importância no cuidado com o diagnóstico diferencial pelo CHIKV e na elucidação do efeito colateral em con-sequência do uso de Hidroxicloroquina.

Palavras-chave: Eritema Multiforme. Chikungunya, Hidroxicloroquina.

\begin{abstract}
The incidence of pharmacodermias is around 5 to $30 \%$, between 3 to $6 \%$ of all hospital admissions. Adverse drug reactions (ADR) are among the leading causes of death in several countries. The objective of this study was to report the case of a patient who presented a clinical features of Erythema Multiforme after managing Hydroxychloroquine in Chikungunya (CHKV) therapy. Subsequently, the case evolved into a generalized exanthem. After the remission of the skin lesions, it was diagnosed as seronegative Rheumatoid Arthritis (RA). The corticoid and methotrexate therapy was continued and there was a significant improvement of the patient's health condition. This study concluded the importance for CHIKV differential diagnosis care and in the elucidation of the side effect as a consequence of Hydroxychloroquine use.

Keywords: Erythema Multiforme. Chikungunya. Hydroxychloroquine.
\end{abstract}

\section{Introdução}

As farmacodermias são doenças tegumentares (pele e/ou mucosa) e/ou sistêmicas desencadeadas, direta ou indiretamente, de diferentes maneiras, pelo uso de fármacos. Etimologicamente, o conceito de Farmacodermia não necessariamente condiz com o termo em si, de maneira que talvez fosse melhor designála como reações medicamentosas adversas (RMA) ${ }^{1,2}$.

Habitualmente é difícil precisar a verdadeira incidência das RMA. Estudos estimam que as RMA representem de 3 a $6 \%$ de todas as admissões hospitalares. Dos doentes hospitalizados, 5 a $30 \%$ apresentam farmacodermias; e em consequência da hipersensibilização, $30 \%$ dos casos podem adquirir novas RMAs. Em determinados hospitais, 1/7 do tempo de internação é consumido por este tipo de doente. As RMA estão entre as principais causas de morte em vários países ${ }^{3}$.

As RMA são menos frequentes em crianças (imaturidade imunológica e ausência de exposição prévia), com uma incidência estimada em torno de $1,5 \%$, que aumenta com a idade. Apesar da falência imunológica relativa, as RMA prevalecem no idoso em função das alterações relacionadas com a farmacocinética dos medicamentos e com o uso de múltiplos fármacos. Mulheres são mais frequentemente acometidas. O clima e o ambiente têm importância no desencadeamento de determinadas farmacodermias como na foto sensibilização ${ }^{4}$. Ademais, há fatores que predispõem ao aparecimento de RMA, como ocorre nos casos de substâncias indutoras de lúpus eritematosos, deficiência genética de enzimas e alelos HLA de classe 
I e II.

Dentre as RMA, as causas de Eritema Multiforme (EM) Minor são infecções (herpes, Mycoplasma pneumoniae, influenza, histoplasmose). Em contrapartida, as causas de EM Major possuem uma etiologia medicamentosa, nas quais são mais comuns por sulfas, penicilinas, anticonvulsivantes aromáticos, alopurinol e anti-inflamatórios não hormonais (AINEs) ${ }^{4}$. Entretanto, o caso relatado evidencia uma relação da manifestação com o uso de hidroxocloroquina e a ocorrência de uma erupção eritematosa de início súbito, distribuição assimétrica e pruriginosa. Assim, os relatos de novos casos com manifestações etiológicas distintas das RMA são de extrema importância no desenvolvimento, controle, prevenção e tratamento destas reações, visto que podem ser letais.
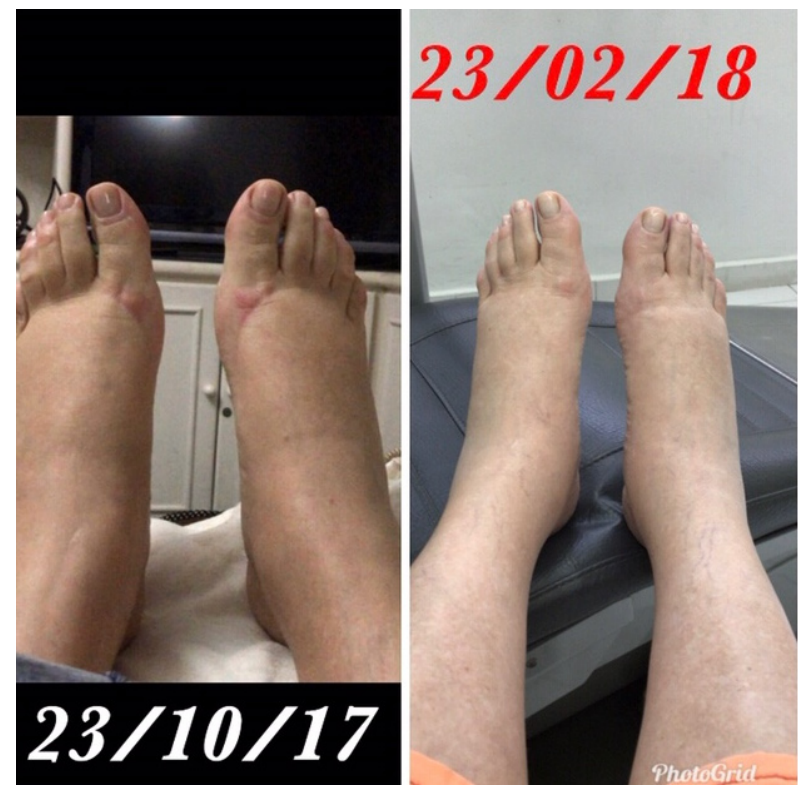

Figuras 1 e 2. Evolução com edema de membros inferiores. Fonte: acervo pessoal do paciente.

\section{Relato de Caso}

Este estudo teve parecer aprovado de número 3.328.085/2019-CEP/Universidade de Vassouras. Paciente, 54 anos, feminina, branca, em setembro de 2017, deu entrada na emergência, com queixa de edema nos membros superiores e inferiores (Figuras 1 e 2) acompanhadas de artralgia com parestesia nas metacarpos falangianas há duas semanas. Inicialmente suspeitou-se de síndrome do túnel do carpo STC e foi prescrito AINEs.

Paralelamente, apresentou diagnóstico de "encurtamento das fibras miofasciais", e fez fisioterapia, que segundo a paciente agravaram a dor na palma das mãos com sensação de "perfuração".

A reumatologia solicitou 20 reações sorológicas, em 13/11, e os resultados apresentaram sorologias negativas, exceto, IgM REAGENTE para Chikungunya CHIKV. Assim, prosseguiu o protocolo de tratamento com prescrição de Hidroxicloroquina $400 \mathrm{mg} \mathrm{VO}$ $1 \mathrm{cp} /$ dia por 30 dias. Em 09/12, há 5 dias do término do tratamento, a medicação (cloroquina) foi suspensa devido ao aparecimento de lesões eritematosas com halo periférico caracterizando as lesões em alvo sem prurido nos membros superiores, tronco e tórax (Figura 3 e 4).

Contudo, após dois dias o quadro se intensificou, e a paciente foi internada com diagnóstico de Eritema Multiforme por Hidroxicloroquina. A mesma permaneceu internada por cinco dias fazendo uso de Hidrocortisona 500mg IV 6/6h. O exame clínico

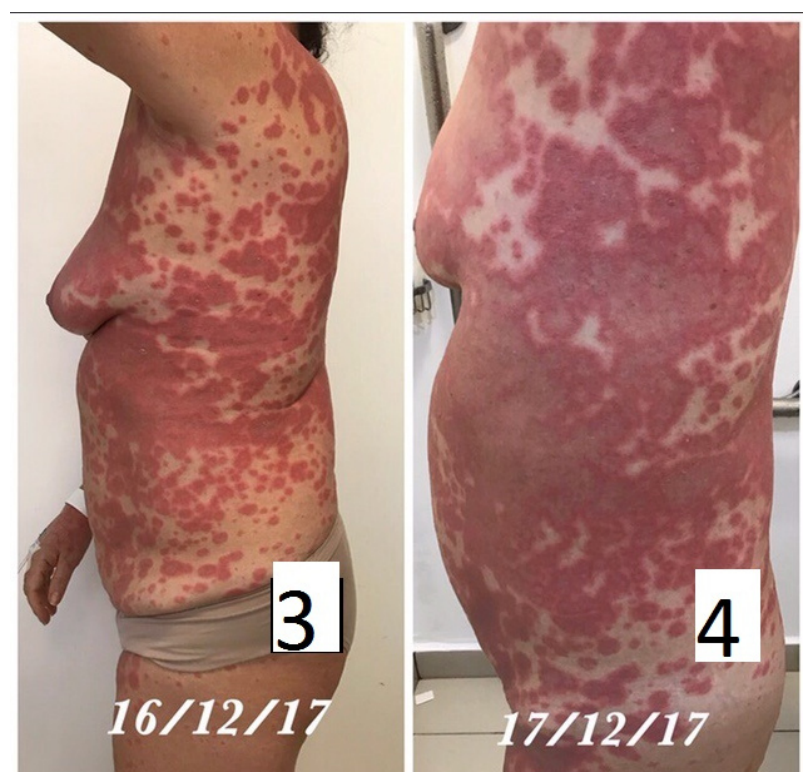

Figuras 3 e 4. Tronco dorsolateral, região abdominal e das mamas de acordo com a progressão das lesões.

Fonte: Acervo pessoal do paciente.

apresentou exantema disseminado, algumas lesões em placas, e outras escamosas com exulcerações, principalmente nas extremidades superiores, tronco, cabeça e face (Figura 5).

Após a alta hospitalar manteve-se corticoideoterapia, e em 02/2018 a infectologia emitiu parecer negativo de contato prévio para o vírus Chikungunya. Logo, o exame clínico confirmou o diagnóstico de Artrite Reumatóide soronegativa e iniciou-se o tratamento com Metotrexato e Vitaminas (B12, ácido fólico) para suporte.

Atualmente a paciente encontra-se assintomática, em bom estado geral, sem complicações e acompanhamento periódico pela reumatologia. 


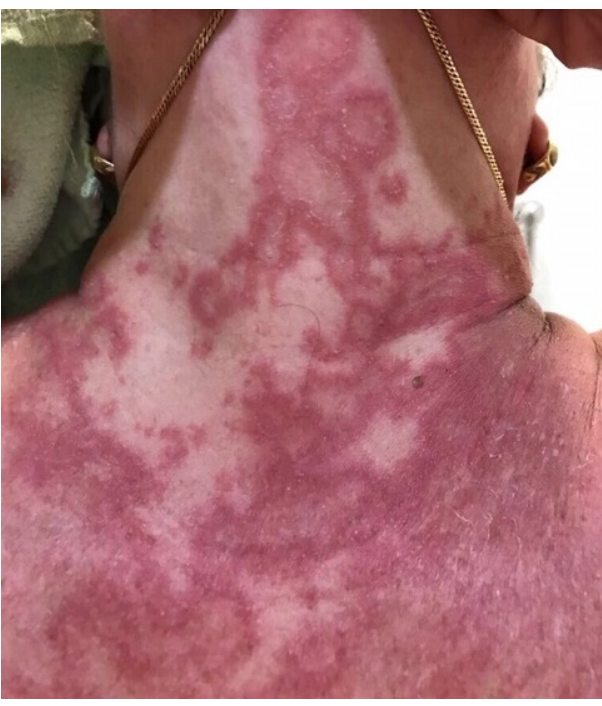

Figura 5. Tronco e pescoço. Fonte: Acervo pessoal do paciente.

\section{Discussão}

As causas de Eritema Multiforme envolvem hipersensibilidade às drogas, infecções virais e estreptocócicas, entre outras. Constitui-se em uma síndrome que se caracteriza por lesões polimorfas: lesões em alvo, em concomitância ou não, lesões purpúricas e bolhas, principalmente em dorso do tórax e em extremidades ${ }^{3}$. Estas reações assemelham-se ao caso relatado neste estudo, contudo, não ocorreu o acometimento mucoso, oral, genital ou oftálmico, conhecida como Eritema Polimorfo major ou Síndrome de Stevens-Johnson ${ }^{4}$.

Dentre as farmacodermias, o Eritema Multiforme constitui-se em uma das reações cutâneas medicamentosas que possuem maior risco à vida. A doença está relacionada a uma série de drogas, como alopurinol, anticonvulsivantes, barbitúricos, cefoxitina e hidroxicloroquina.

O diagnóstico diferencial se faz com: dermatite de contato generalizada, psoríase pustulosa, eritemas figurados, pênfigo foliáceo, entre outras. Porém neste caso, o diagnóstico foi baseado apenas na apresentação clínica e na prova terapêutica com a suspensão da hidroxicloroquina e regressão das lesões, pois não foi possível a realização da biópsia e exame histopatológico.

O diagnóstico da febre Chikungunya que fora levantado, no caso, de forma equivocada é tipicamente clínico, uma vez que a associação de febre aguda com artralgia e/ou artrite intensa de instalação aguda é altamente sugestiva, com elevada sensibilidade e valor preditivo positivo, em áreas onde a doença é endêmica e onde há epidemias ${ }^{5,6}$. Atualmente, no Brasil, a recomendação do Ministério da Saúde é usar os critérios clínico-epidemiológicos para definição de caso suspeito de febre Chikungunya. No entanto, houve controvérsia quanto à utilização de hidroxicloroquina, baseada nos resultados da primeira sorologia, pois os demais exames foram não reagentes para CHIKV.

As evidências existentes sobre o uso de antimaláricos na fase subaguda dos sintomas refratários a analgésicos são escassas e apontam para uma resposta clínica pouco significativa ${ }^{7,8}$. Um estudo indiano comparou um grupo de pacientes com tempo de doença mínimo de 30 dias (tempo médio de nove semanas) com cloroquina $(250 \mathrm{mg} / \mathrm{dia})$ com outro grupo com meloxicam $(7,5 \mathrm{mg} / \mathrm{dia})$ durante 24 semanas. Embora a resposta tenha sido numericamente melhor no grupo com cloroquina, não houve diferença estatisticamente significativa na melhoria da escala visual analógica de dor e número de articulações dolorosas 9 .

O risco das complicações nos pacientes com estas reações cutâneas é relevante, visto que necessitou de internação hospitalar e observação clínica por cinco dias, corticoide terapia EV em dose máxima diária, o que demonstra a importância deste estudo e gravidade do caso apresentado. O número de reações medicamentosas adversas é crescente, assim, torna-se necessário o conhecimento sobre o diagnóstico, manejo e tratamento destas para prevenir piores desfechos.

\section{Referências}

1. Barbaud A. Drug patch tests in the investigation of cutaneous adverse drug reactions. Ann Dermatol Venerol. 2009; 36 (8-9): 635-44.

2. Rubem DA, Azulay L, Azulay A. Farmacodermias. In: autor do livro. Dermatologia, 6. ed. Rio de Janeiro: Guanabara Koogan, 2015; p. 223-233.

3. Cheung MJ, Taher M, Lauzon GJ. Acneiform facial eruptions: a problem for young women. Can Fam Physician. 2005; (51): 527-33.

4. Yuge S, Romanini AM, Silveira-Souza D, Gonçalves CB, Martelli ACC. Departa-mento de medicina cutânea e interna. An Bras Dermatol 2005; 80 (Supl 2): S77-188.

5. Sissoko D, Ezzedine K, Moendandze A, Giry C, Renault P, Malvy D. Field evalua-tion of clinical features during chikungunya outbreak in Mayotte, 2005 $\square 2006$. Trop Med Int Health 2010; 15: 600-607.

6. Conselho Federal de Medicina - Febre de Chikungunya $\square$ manejo clínico. 2015. http://portal.cfm.org.br/index.php?option=com content\&view $=$ article\&id $=25398: 2015 \square 03 \square 16 \square 17 \square 58 \square 53$ \&catid=3[Acesso $17 / 09 / 2016]$.

7. Macpherson C, Noel T, Fields P, Jungkind D, Yearwood K, Simmons M, et al. Clin-ical and serological insights from the Asian lineage chikungunya outbreak in Grenada, 2014: an observational study. Am J Trop Med Hyg. 2016; 95: 890-893.

8. Chopra A, Saluja M, Venugopalan A. Effectiveness of chloroquine and inflammatory cytokine response in patients with early persistent musculoskeletal pain and arthritis following chikungunya virus infection. Arthritis Rheumatol. 2014; 66: 319-326.

9. Padmakumar B, Jayan JB, Menon RMR, Krishnankutty B, Payippallil R, Nisha RS. Comparative evaluation of four therapeutic regimes in chikungunya arthritis: a prospec-tive randomized parallel $\square$ group study. Indian J Dermatol. 2009; 4: 94-101.

10. Silva L, Roselino AM. Reações De Hipersensibilidade A Drogas (Farmacodermia). RMRP [Internet]. 30dez.2003 [citado 8 fev.2019]; 36(2/4):460-71. Available from: http://www.journals.usp.br/rmrp/article/ view/29419. 